\title{
Child and Adolescent Psychiatry for the Future General Adult Psychiatrist
}

Peter Hill, Senior Lecturer, and David CotTrell, Lecturer, Child \& Adolescent Section, Department of Psychiatry, St George's Hospital Medical School, Tooting, London SW17

It is an expectation of the Royal College of Psychiatrists that its members should demonstrate a knowledge of the psychiatry of childhood and adolescence. Prospective members are examined on this and a clinical placement in child and adolescent psychiatry is a recognised specialty component of general training. That this is so reflects the fact that one quarter of the population is composed of individuals under the age of 18 . This group has an appreciable psychological morbidity rate with patterns of psychological disability and suffering which differ appreciably from those encountered in general adult practice. A competent general psychiatrist should be familiar with the pattern of psychiatric pathology is childhood and be conversant with the services available for its treatment. For the health of the psychiatric profession as a whole, it is important that trainees have some working experience of child and adolescent psychiatry so that they may find whether this area of practice is attractive to them. Experience at registrar level provides an important recruitment opportunity for future senior registrars and thus consultants in child and adolescent psychiatry. Successful completion of general training in psychiatry with possession of membership of the Royal College of Psychiatrists is effectively a universal prerequisite for entering the senior registrar grade and higher training in child and adolescent psychiatry.

Nevertheless, most psychiatrists in general training will not become child and adolescent psychiatrists. Of what value is a child and adolescent psychiatry placement to them over and above the help it may give them in passing the Membership exam as a hurdle in their chosen career of adult psychiatrists, either general or in one of the adult sub-specialties?

Suggested aims and objectives of clinical training in child and adolescent psychiatry

1. The ability to take a developmental perspective in formulation

Young people are self-evidently passing through a phase of rapid growth in both physical and psychological terms. The developmental parameter is crucial for formulation in child and adolescent psychiatry and is of equal importance in some areas of adult psychiatry such as the assessment and treatment of personality disorders or the management of psychiatric disorder in the late teenage years. The issues of dependency and the pathology of attachment are relevant in adult psychotherapy, marital therapy, and the management of disability at any age, particularly with respect to accelerated dependency in dementia. The very necessity of addressing these issues in child and adolescent psychiatry means they cannot be overlooked so the registrar in training must address them in the management of his/her patients. A variant is the development of an understanding of how adversity and life events impinge upon children. Such understanding is relevant to formulating how earlier life experience has created vulnerability or resilience in the psychological development of a patient presenting in adult life.

2. Interviewing the developmentally young and their families The skills required for direct interviewing with young people include elements that are appropriate in adult psychiatry. Diagnostic interviewing of the older yet immature teenager or the mentally handicapped adult will require a modification of technique in order to account for emotional or cognitive immaturity. The same point also applies to counselling. If a general adult psychiatrist is to assess the impact of psychiatric disorder in a parent upon their children or to advise in custody access decisions, knowledge of how to interview children is necessary.

In similar vein the skills required to interview several members of a family together, a practice common in child and adolescent psychiatry, has clear implications for family or marital work in adult psychiatry.

3. Operating within a family context

It is a byword of child and adolescent practice that family issues are central to formulation and treatment. This would appear to be only too often a neglected area in adult psychiatric practice wherein close relatives may only be seen as possible sources of information about the patient or as somewhat peripheral accessories to psychiatric management. Given current models of psychiatric disorder in childhood, it becomes impossible to ignore family relationship issues in the management of disturbed children and the obligatory nature of this forces the registrar in training to confront these issues which might otherwise be avoided. Particular emphasis can be placed upon two issues: firstly the close and important relationship between parental psychiatric disorder and childhood disturbance, a matter which should unite the clinical practice of adult and child psychiatrists more than it does; secondly the studies on family relationships and the course of schizophrenia which have led to experimental family interventions using techniques comparable to those already well exercised in child and adolescent psychiatry. 


\section{The relevance of social issues}

The increased prevalence of childhood psychiatric disorder in inner city areas has forced child and adolescent psychiatrists actively to consider social issues in both academic and clinical arenas. Social adversity exercises a constraint upon family functioning through a variety of mechanisms which must be explored in clinical management. At the same time the influences of school and peer group have been increasingly appreciated as elements to be assessed in formulation and treatment. Social management by environmental manipulation is a valid treatment option in child and adolescent psychiatry.

For such reasons, a trainee working in a child and adolescent psychiatric service will gain experience in taking a social perspective on individual psychopathology which would sensibly broaden his or her approach to adult patients in general psychiatry.

\section{Reliance upon psychological methods of treatment}

Child and adolescent psychiatry as a service is a sparse user of medication. Reliance is placed on a variety of psychological managements, from advice and environmental manipulation at one end to in-depth individual psychotherapy utilising a detailed model of the unconscious and transference issues at another. The vast majority of child psychiatry clinics would rely on a range of treatment methods within which the trainee has to acquire some personal experience and expertise without being able to reach too readily for the prescription pad.

6. Familiarisation with the child and adolescent psychiatry service

Several aspects of child and adolescent psychiatry can cause trainees to reassess their habitual modes of thought. For instance, only a few psychiatrically disordered children are admitted to hospital; most are managed within a family setting in their homes with out-patient and telephone contact. The psychiatrist finds himself directly involved with the child and family without nursing staff as an intermediary and has to intervene at all levels of complexity from giving straightforward advice or information about ordinary parenting to more sophisticated interventions.

\section{Consideration of the wider system}

Children's services form a pattern of agencies, each with a different stake in the welfare of their clients. The successful management of psychiatric disorder in childhood often involves liaison with a number of medical and community agencies to ensure their efforts are brought to bear or alternatively, to avoid duplication. This, for the trainee, means that liaison skills must be learned which do not depend on medical hierarchy, that issues of confidentiality have to be addressed, that too narrow a 'medical model' destroys professional credibility and so forth. The appreciation of the contributions of various agencies in the management of disorder means that some thought needs to be given to the operation of systems and institutions other than hospitals.

\section{Multidisciplinary teamwork}

Within the domain of child and adolescent psychiatry medical expertise is not always pre-eminent and the relative contribution of non-medical disciplines is often more cogent than is the case in general adult psychiatry. The experience of working alongside senior and experienced non-medical colleagues in collaborative therapy or cotherapy can hardly be avoided on a clinical child and adolescent psychiatry placement.

\section{Interactions with physical problems}

A competent child and adolescent psychiatry service will attract referrals from paediatricians and would ordinarily operate a systematic paediatric liaison service. Involvement of the registrar in such arrangements allows them to perceive the interaction of biological, individual psychological and family issues. A substantial amount of paediatric morbidity derives from just such interplay and is frequently responsive to psychological interventions in a manner which provides a clear illustration of principles which can be applied to abnormalities of illness behaviour in adults.

\section{Structure and supplementary experience within a placement} The above considerations could be highlighted as what a general trainee might expect to obtain from a clinical placement in child and adolescent psychiatry.

One way of formalising their relevance for future general general psychiatrists might be to prepare a checklist for trainees entering a placement so that cases can be selected to ensure they gain experience in each of the above areas.

They could be supported by a programme of seminars and visits in order to add emphasis. The seminars might follow the topics above though, for example, the issue of psychosocial development could be restricted to considerations of moral development or interpersonal development on which there are suitable review papers which are relevant not only to children but also to adults with immature personalities.

Recommended visits could be undertaken by registrars who are in child and adolescent psychiatry placements. This could enhance their clinical practice in placement by offering an opportunity to familiarise themselves with ordinary children and their school settings. A possible programme of visits would be as follows:

(1) a paediatric child development clinic;

(2) a local authority day nursery or a pre-school playgroup;

(3) an ordinary primary school;

(4) an ordinary secondary school;

(5) a community child guidance clinic;

(6) a visit to an adolescent psychiatric unit;

(7) a visit to a school for autistic children or a similar school catering for a selective developmental disability.

It might also stipulate that any registrar on placement should:

(1) see at least one supervised case in family therapy;

(2) should undertake at least one home visit on which a report should be written and scrutinised; 
(3) should be videoed at least once interviewing a child or young teenager;

(4) should go at least one social services conference (e.g. on child abuse).

A possible extension of the principle to make the links with general psychiatry more tangible would be for the trainee to write up one case of an adult patient on their next clinical placement in child psychiatry terms i.e. with full formulation of development and family factors. This could then be jointly discussed between the trainee, their previous supervising consultant in child and adolescent psychiatry and the consultant responsible for the adult patient.

\section{The First Year's Operation of a Regional Drug Problem Team}

Philip M. Fleming, Consultant Psychiatrist and Director, Wessex Regional Drug Problem Team, Northern Road Clinic, Cosham, Portsmouth

The recent announcement of a further $£ 5$ million which has been distributed to Regional Health Authorities' makes a total of over $£ 22$ million that has been made available specifically for the development and expansion of services for drug misusers over the past three years. An important element in the development of such services has been the formation of Regional Drug Problem Teams; this seemed a useful time to review the working of one such team over the past year.

\section{Setting up the team}

The Wessex Regional Health Authority produced its policy for services for drug misusers in April $1985^{2}$ after wide consultation and after receiving advice from a steering group convened for this purpose. Amongst the proposals was one to establish a Regional Drug Problem Team (RDPT). This was to have three functions: (1) To advise and encourage Districts in developing their local services, advise on clinical policies and case management, set standards, provide and co-ordinate training programmes. (2) Where needed, and until District expertise has grown, to advise in specific diffcult cases. (3) In the longer term to provide specialist advice, information collection, monitoring and evaluation of services to Districts and to the Region. In practice the RDPT was to act as a liaison agency for all organisations in the Region concerned with drug misuse. These are largely the functions for a Regional Drug Problem Team that were first set out in the Treatment and Rehabilitation Report of the Advisory Council on the Misuse of Drugs. ${ }^{3}$

Wessex Region is made up of 10 Districts and has a total population of 2.8 million. It includes the counties of Hampshire, Wiltshire, Dorset and the Isle of Wight and parts of Avon. The main centres of population are Bournemouth, Southampton and Portsmouth in the south, Swindon and Basingstoke in the north and the cathedral cities of Winchester, Salisbury and Bath; the rest of the region is mostly rural. At the time of the Regional Report in April 1985 only Portsmouth, Bournemouth and Southampton had any services for drug misusers. As the service in Portsmouth was the most comprehensive ${ }^{4}$ it was decided to base the RDPT there. The team is made up of a representative of Hampshire County Council Social Services Department, who works as a Principal Officer with that Department as their adviser on addictions and has had many years' experience in the field; a full time community psychiatric nurse at clinical manager level who had had previous experience in the Portsmouth Drug Service; the author for two sessions a week as psychiatrist and director of the team. There was in addition part-time clerical support. The team shared office space with the Portsmouth staff for the first nine months of its existence but now has separate offices for itself.

\section{Planning and development of services}

The first task of the team was to meet those staff in Districts responsible for overseeing the development of services for drug misusers. This was normally the District Medical Officer-although these posts were often disappearing with re-organisation, and it was not always clear who would ultimately be responsible for drug services. One of the early requirements of Districts was to set up a District Drug Advisory Committee. These multidisciplinary groups (also proposed in the Treatment and Rehabilitation Report) are charged with monitoring drug problems in Districts and assessing and planning services. Where possible we have tried to attend meetings of these committees. In those Districts where services already existed or where personnel had been identified to start a service, we met the people actually concerned.

In these early meetings we were able to assist Districts in formulating their plans for services. We often found that Districts did not think they had much of a drug problem, and there was frequently scepticism about the need to develop specific services for drug misusers. I heard this view voiced by more than one Division of Psychiatry. Some Districts had tried to make some assessment of the incidence of drug misusers, although none used the strategies recommended by the Drug Indications Project. ${ }^{5}$ Our advice was 